\title{
Ejecución de Talleres de Tesis y su Influencia en el Fortalecimiento de Habilidades Investigativas de los Estudiantes de Posgrado de Apurímac - 2015
}

\section{Running theses workshops on strengthening research skills of students pos degree of Apurimac - 2015}

\author{
Antonia Mosqueira
}

\section{RESUMEN}

La presente investigación tuvo como objetivo analizar y explicar las diferencias significativas entre el grupo de control y el grupo experimental respecto al nivel de fortalecimiento de las habilidades investigativas de los estudiantes de posgrado en Pedagogía de educación superior después de haber participado en talleres de tesis, dicha investigación tiene un enfoque cuantitativo, diseño experimental y tipo explicativo. La muestra conformada por 70 estudiantes de maestría en pedagogía superior de una universidad privada de la ciudad de Abancay. Para el trabajo de campo los instrumentos usados fueron guía de observación, cuestionario y prueba educativa.

El estudio tiene relevancia socio educativo ya que beneficia a maestrantes de distintas profesiones que ejercen la docencia universitaria, por la necesidad de elaborar proyectos de investigación y la misma tesis. Se llegó a la conclusión: la ejecución de talleres de tesis influye de forma determinante en el fortalecimiento de habilidades investigativas.

Palabras clave: Desempeño, docente-asesor, talleres, tesis, habilidades, investigativas

\section{ABSTRACT}

This research aimed to analyze and explain the significant differences between the control group and the experimental group compared to the level of strengthening research skills of graduate students in Pedagogy of higher education after participating in workshops thesis that research has a quantitative approach, experimental design and explanatory type.

The sample consists of 70 master's students in higher education at a private university in the city of Abancay. Fieldworks for the instruments used were observation guide, questionnaire and educational test. The study has relevance and educational partner benefits Grandee of different professions exercising university teaching, by the need to develop research projects and the same thesis. He concluded: the implementation of theses workshops have a decisive bearing on strengthening research skills.

Keyword: senior teacher performance, running workshops and research skills. 


\section{INTRODUCCIÓN}

La investigación es una práctica reflexiva, de la mano de un experto que orienta y transmite las competencias necesarias en el hacer.

Sin embargo desde la perspectiva pedagógica didáctica es poco lo que se sabe sobre la formación de investigadores.

Es por ello la preocupación que lleva a indagar sobre las prácticas de enseñanza y aprendizaje en la formación de investigadores en programas de maestría (en la región de Apurímac) y que, los docentes de metodología de la investigación tengan nuevas y mejores herramientas que les permitan alcanzar los objetivos deseados.

Fernández, Cruz y Wainerman consideran que ambientes de aprendizaje critico natural que promuevan el aprendizaje situado a partir de la participación en un contexto en el que bajo la guía de los docentes, se desarrollan tareas propias de la comunidad académica teniendo como resultado tanto el aprendizaje del quehacer de la investigación como los modos de escritura académica, los valores de la comunidad académica y el rol crítico del trabajo de otros.

El objetivo general del estudio: Analizar y explicar los hallazgos de las diferencias significativas entre el grupo de control y el grupo experimental respecto al nivel de fortalecimiento de las habilidades para investigar, después de haber participado en talleres de tesis, en los resultados de la post prueba de los estudiantes del III ciclo de la maestría en educación con mención en pedagogía en educación superior de la Universidad Tecnológica de los Andes de la ciudad de Abancay durante el año 2015.

\section{Objetivos específicos}

Describir el incremento significativo en el nivel de habilidades para investigar de acuerdo al resultado de las pruebas de pre y post aplicadas al grupo de control.

Argumentar el incremento significativo en el nivel de habilidades para investigar de acuerdo al resultado de las pruebas de pre y post aplicadas al grupo de experimento.

Hipótesis general de la investigación:

Se observa un incremento significativo en el fortalecimiento de las habilidades para investigar del grupo experimental frente al grupo de control después de haber participado en talleres de tesis, en los resultados de la post prueba de los estudiantes del III ciclo de la maestría en educación con mención en pedagogía en educación superior de la Universidad Tecnológica de los Andes de la ciudad de Abancay.

La ejecución de talleres, está definido, como una realidad integradora, compleja reflexiva, en que se unen la teoría y la práctica como fuerza motriz del proceso pedagógico, orientado a una comunicación constante con la realidad social y como un equipo de trabajo altamente dialógico formado por docentes y estudiantes, en el cual cada uno es un miembro más del equipo y hace sus aportes específicos.

La ejecución de talleres debe contar con una buena metodología: centrada en el estudiante con una comunicación horizontal y un adecuado material: innovador, motivador y operativo.

Según Egg, los principios pedagógicos del taller son:

Es un aprender haciendo porque los conocimientos se adquieren en una práctica concreta, se aprende haciendo.

Es una metodología participativa porque se requiere de la participación activa de todos los integrantes del taller, para ello se deben desarrollar actitudes y compartimientos participativos. 
Es una pedagogía de la pregunta, contrapuesta a la pedagogía de la respuesta propia de la educación tradicional, es decir según la pedagogía tradicional es algo que puede depositarse en el otro, pero no es la pedagogía propia del taller, en donde el conocimiento se produce fundamentalmente y casi exclusivamente en respuesta a preguntas.

Es un entrenamiento que tiende al trabajo interdisciplinario y al enfoque sistémico es decir el taller tiende a la interdisciplinariedad, en cuanto es un esfuerzo por conocer y operar, asumiendo el carácter multifacético y complejo de toda realidad: la realidad nunca se presenta fragmentada de acuerdo a la clasificación de las ciencias o la división de las disciplinas académicas, sino que está todo interrelacionado.(3)

La relación docente/alumno queda establecida en la realización de una tarea común, el alumno se inserta en el proceso pedagógico como sujeto de su propio aprendizaje, con la apoyatura teórica y metodológica de los docentes y de la bibliografía y documentación de consulta que las exigencias del taller vayan demandando.

Carácter globalizante e integrado de su práctica pedagógica, taller crea un ámbito y las condiciones necesarias para desarrollar la unidad de enseñanza aprendizaje, además, ayuda a superar las dicotomías entre teoría y práctica, educación y vida, el conocer y el hacer

Implica y exige de un trabajo grupal y el uso de técnicas adecuadas, es necesario utilizar técnicas grupales que potencien el trabajo en esta modalidad.

Habilidades Investigativas según Machado y Montes de Oca (2009, pág. 34) constituyen eje transversal de la formación investigativa:

El desarrollo de habilidades investigativas es una de las vías que permite integrar el conocimiento a la vez que sirve como sustento de autoaprendizaje constante; no solo porque ellas facilitan la solución de las más diversas contradicciones que surgen en el ámbito laboral y científico, sino además porque permiten la autocapacitación permanente y la actualización sistemática de los conocimientos, lo cual es un indicador de competitividad en la época moderna.

Clasificación de la Habilidades investigativas según Libertad (2003):

1. Habilidad para la obtención del conocimiento científico (Dimensión)

Indicadores:

-Delimitación del problema y su justificación.

-Planteamiento del problema de la investigación.

-Elaboración del marco teórico y conceptual.

-Formulación de los objetivos.

-Formulación de hipótesis de investigación.

-Proceso de operacionalización de las hipótesis.

-Diseño de técnicas de recolección de datos.

Aplicación en el trabajo de campo.

-Selección de técnicas de procesamiento estadístico.

-Análisis e interpretación de la información.

2. Habilidad para la presentación y discusión de resultados (Dimensión)

Indicadores:

-Elaboración de informes del trabajo científico.

-Presentación y discusión ante el tribunal de los informes y resultados.

-Divulgación de los resultados. 
3. Habilidad inicial para la evaluación de la investigación (Dimensión)

Indicadores:
-Identificar resultados relevantes.

-Detectar los principales logros y dificultades del trabajo investigativo.

-Identificar líneas de investigación y tipos de diseños metodológicos.

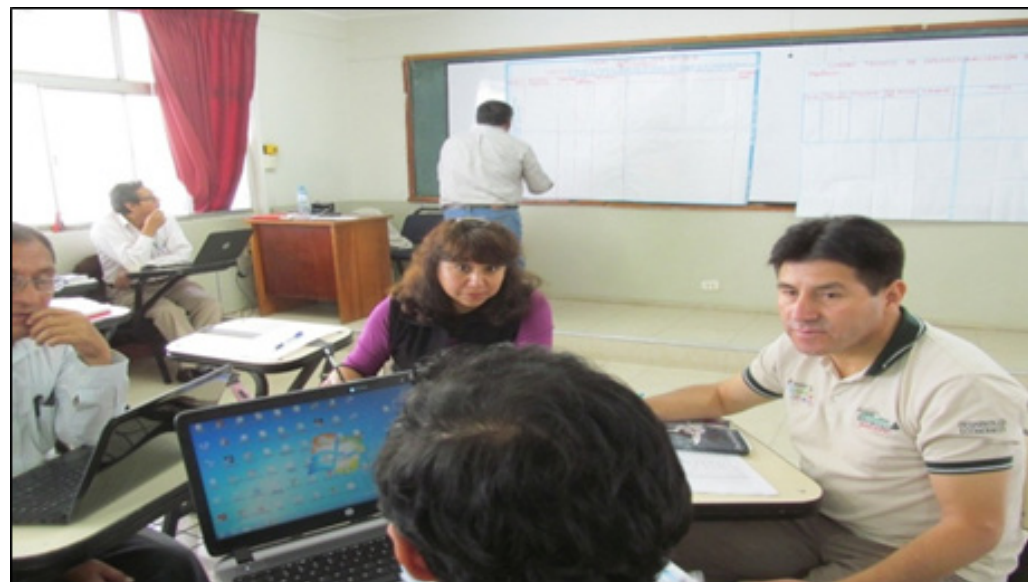

Fuente: Imagen tomada por la autora

\section{MATERIAL Y MÉTODO}

\section{Material}

Se realizó una exhaustiva revisión de libros, revistas, artículos, tesis y otros documentos relacionados al tema de investigación.
Para la variable independiente se usó guía de observación y un cuestionario, mientras que para la variable dependiente se usó una prueba educativa, todos los instrumentos fueron aplicados a los estudiantes del III ciclo de la maestría en educación de la Universidad Tecnológica de los Andes, Abancay, 2015.

\section{Tabla $N^{\circ} 1$. Material usado en la investigación}

\begin{tabular}{|c|c|c|c|}
\hline Variable & Instrumento & $\mathrm{N}^{\circ}$ de preguntas & Îtems \\
\hline \multirow[t]{2}{*}{$\begin{array}{l}\text { Ejecución de } \\
\text { talleres }\end{array}$} & $\begin{array}{c}\text { Guía de } \\
\text { observación }\end{array}$ & 52 & $\begin{array}{c}\text { Con frecuencia } \\
\text { A veces } \\
\text { No }\end{array}$ \\
\hline & Cuestionario & 19 & $\begin{array}{c}\text { Con frecuencia } \\
\text { A veces } \\
\text { No }\end{array}$ \\
\hline $\begin{array}{c}\text { Habilidades } \\
\text { investigativas }\end{array}$ & Prueba educativa & 24 & \\
\hline
\end{tabular}




\section{Metodo}

El tipo de investigación cuantitativo, de índole experimental ya que tiene como fundamento que a partir de problemas se genere la proyección de estrategias pedagógicas que son llevadas por medio de talleres y puestas en la práctica con la población de estudio, mediante proceso, las cua- les permiten recopilar información. De acuerdo a los objetivos es aplicativa, porque busca transformar o cambiar la variable Y con la experimentación de $\mathrm{X}$.

Se utilizó el diseño experimental. Se aplicó el pre test y el pos test a dos grupos el grupo experimental y grupo control.

\section{Tabla $N^{\circ} 2$ : Método en la investigación}

\begin{tabular}{cccc} 
Grupos & \multicolumn{3}{c}{$\begin{array}{c}\text { Secuencia de registro } \\
\text { Tratamiento }\end{array}$} \\
& Pretest & Postest \\
Experimental & Y:(habilidades & X: (ejecución & Y: (habilidades \\
(GE) & investigativas) & talleres de tesis) & investigativas) \\
& Y:(habilidades & & Y: (habilidades \\
Control (GC) & investigativas) & & investigativas) \\
& & &
\end{tabular}

\section{Plan de ejecución de los talleres de tesis}

\section{Tabla Nº 3 : Plan de ejecución de los talleres de tesis}

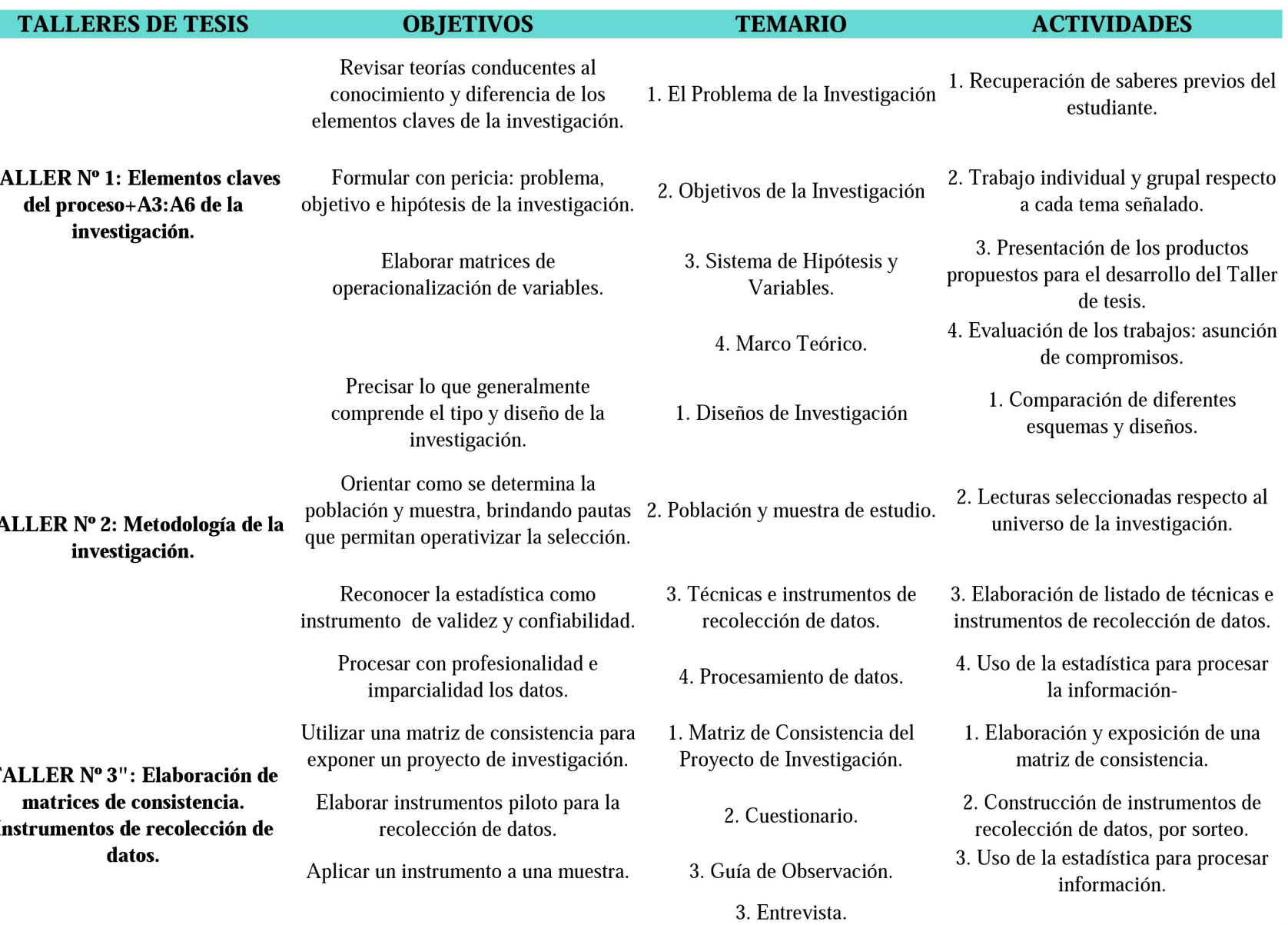




\section{RESULTADOS}

Variable Dependiente: Habilidades Investigativas

Dimensión: Habilidad para la obtención del Conocimiento Científico

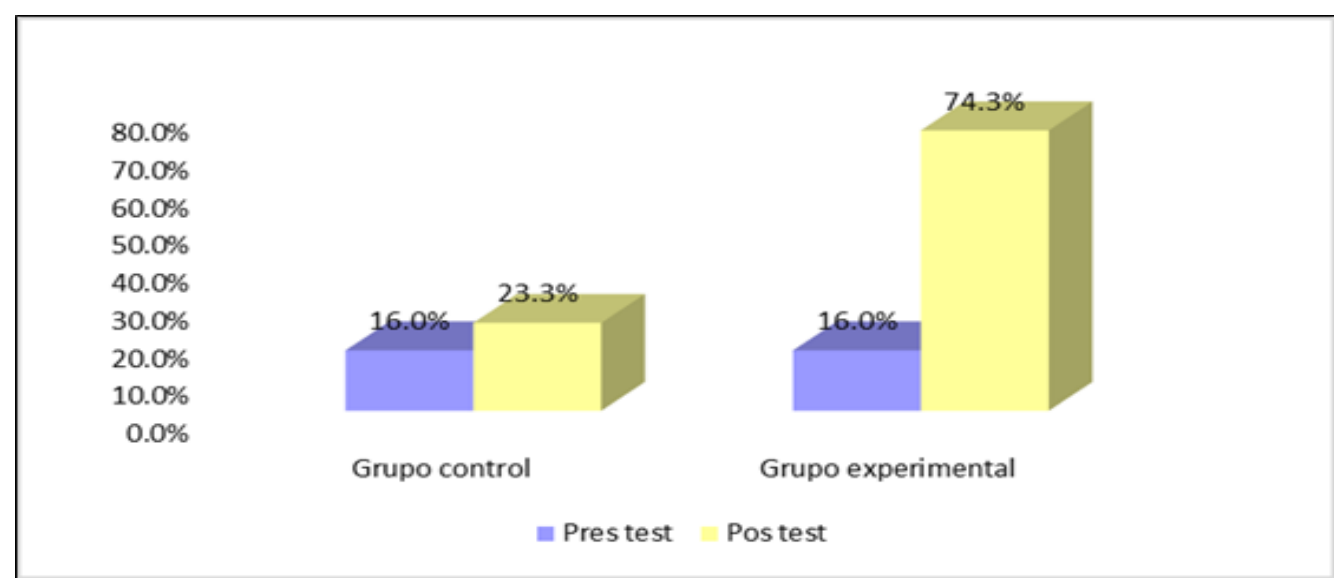

Gráfico 1: Cuadro comparativo de resultados: grupos de control y experimento Fuente: Elaboración propia

\section{Habilidad para la Presentación y Discusión de Resultados}

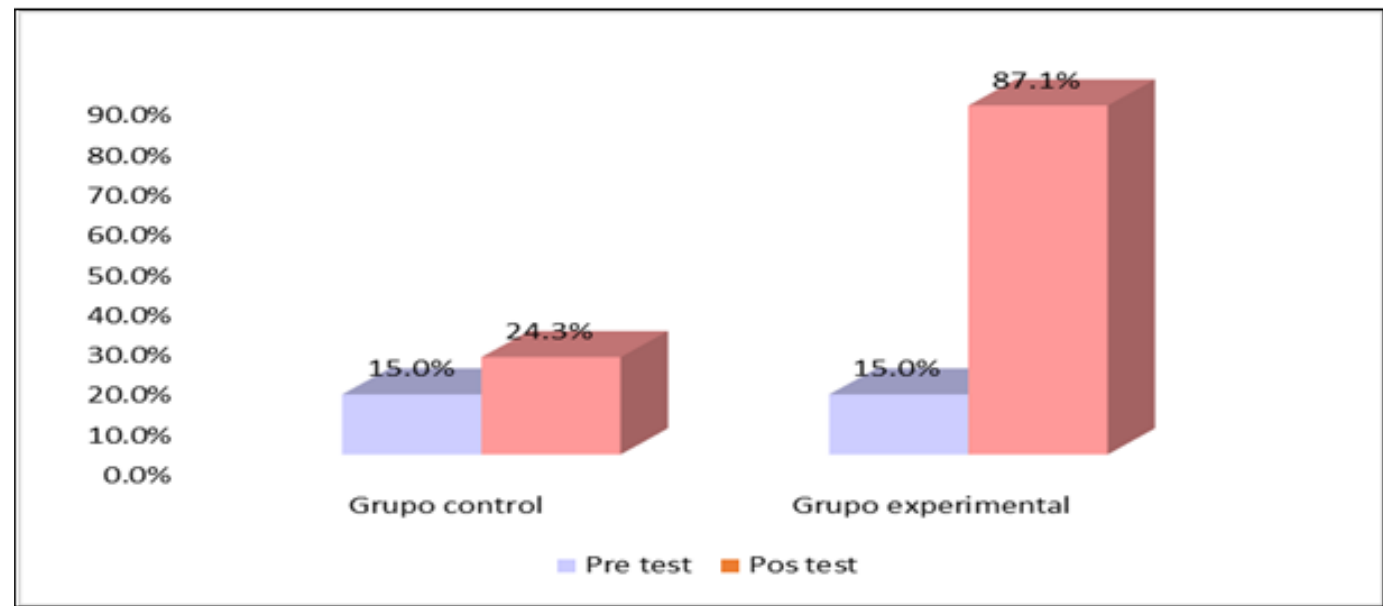

Gráfico 2: Cuadro comparativo de resultados: grupos de control y experimento Fuente: Elaboración propia 


\section{Dimensión: Habilidad inicial para evaluar la investigación}

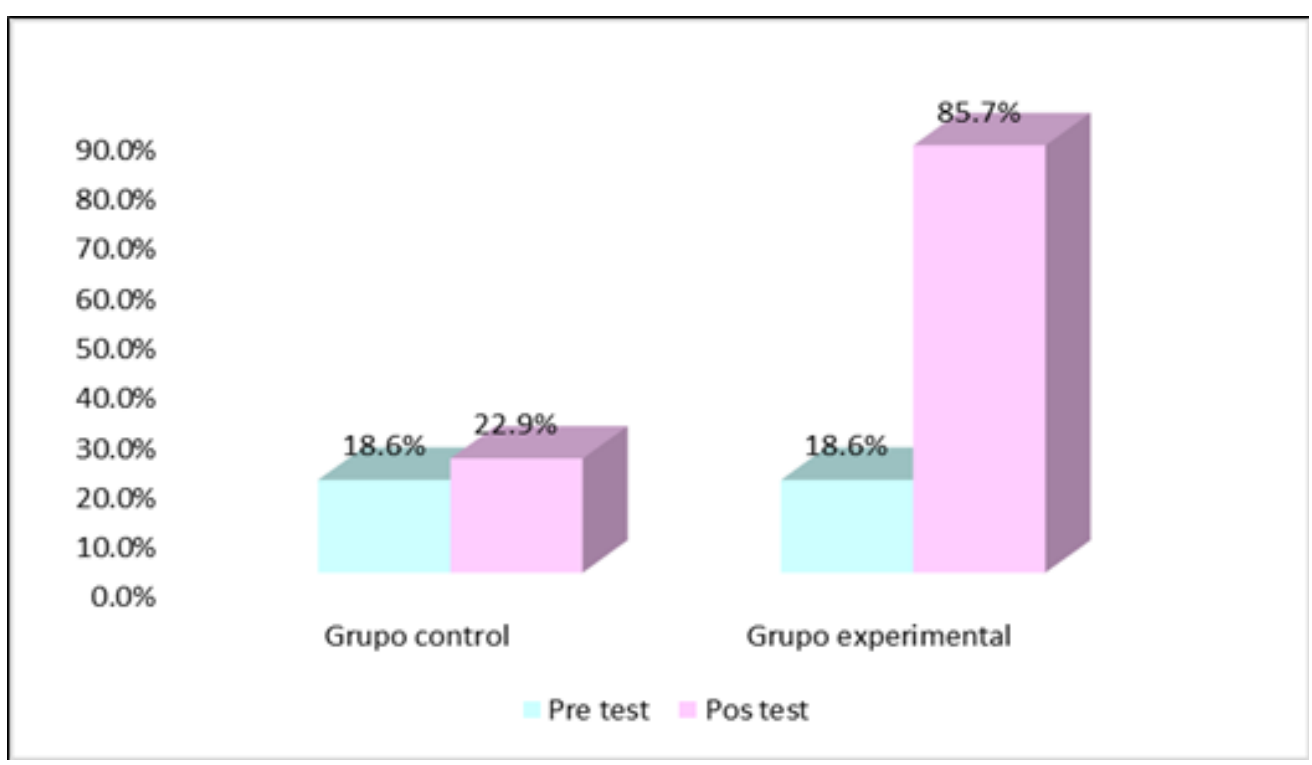

Gráfico 3: Cuadro comparativo de resultados: grupos de control y experimental Fuente: Elaboración propia

\section{DISCUSIÓN}

Al aplicar el estadístico chi-cuadrado de Pearson se obtuvo una probabilidad menor a 0.05 , el cual cae en zona de rechazo, por ello se rechaza la hipótesis nula y se acepta la hipótesis alternativa, a ello se debe agregar que la prueba chi-cuadrado arrojó un resultado de 488.627, con lo que se concluyó que el desempeño docente asesor y ejecución de talleres de tesis influye positivamente en el fortalecimiento de habilidades investigativas de los estudiantes del III ciclo de la maestría en educación con mención en docencia universitaria de la Universidad Tecnológica de los Andes - Abancay, 2015.

Para reforzar este resultado citaremos a Chirino (5) quien define las habilidades investigativas como "el dominio de las acciones generalizadoras del método científico que potencian al individuo para la problematización, teorización y comprobación de su realidad profesional, lo que contribuye a su transformación

Sara Matienzo (6) llegó a la conclusión de que la estrategia metodológica para el desarrollo de habilidades investigativas en los estudiantes permite concebir la diferenciación de las actividades a realizar en sus fases de iniciación, desarrollo y culminación.

Dentro del proceso de enseñanza-aprendizaje muchos docentes implementan los talleres pues consideran que es una buena estrategia pedagógica donde permite que el estudiante pueda desenvolverse e ir entrando más en el tema que está desarrollando. Muchos de estos talleres permiten que los estudiantes puedan desarrollar habilidades investigativas que les ayudarán a definir mejor el rumbo de su investigación y hacer más fácil el proceso de la misma.

\section{Se establece como conclusión}

1.La ejecución de talleres influye positivamente en el desarrollo de habilidades investigativas de los estudiantes de la maestría en educación con mención en docencia universitaria de la Universidad Tecnológica de los Andes - Abancay. Las pruebas estadísticas aplicadas al pre test y pos test confirman lo indicado. 
2. La ejecución de talleres influyen positivamente en la obtención del conocimiento científico. Aquellos alumnos que participaron en el grupo experimental mejoraron su rendimiento ostensiblemente, a diferencia de los que no lo estuvieron. Aplicado el estadístico chi-cuadrado se obtuvo un nivel de diferencia significativo entre dichos grupos.

3. Es posible señalar que existe influencia del desempeño docente asesor y la ejecución de talleres sobre la habilidad para la presentación y discusión de resultados.

4. Respecto al manifiesto de las habilidades investigativas los resultados señalaron que estas varían notablemente en cuanto se ejecute los talleres de manera óptima, utilizando estrategias y material educativo pertinente.

\section{REFERENCIAS}

1. Fernández, L., Cruz, T., \& Wainerman, C. (2014). El taller de tesis como potencial ambiente de aprendizaje situado para la formación en investigación en doctorados en Ciencias Sociales. Rosario: Universidad Nacional de Rosario.

2. Egg, A. (1999). El taller una alternativa de renovación pedagógica. Rio de la Plata: Editorial magisterio.

3. Machado Ramírez, E. F., \& Montes de Oca Recio, N. (2009). Las habilidades investigativas y la nueva Universidad: Terminus a quo a la polémica y la discusión. Humanidades Médicas.

4. Libertad, M. (2003). Formación de habilidades investigativas mediante el programa de maestría en psicología de la la salud. Ciudad de La Habana: Escuela Nacional de Salud Pública.

5. Chirino Ramos, M. V. (2002). Perfeccionamiento de la formación inicial investigativa de los profesionales de la educación. (Tesis de doctorado) La Habana: Instituto Superior Pedagógico Enrique José Varona.

6. Matienzo, S. (2009). Estrategia metodológica para el desarrollo de habilidades investigativas en los estudiantes de Derecho vinculado a la universalización. (Tesis de maestría) Cuba: Universidad de Matanzas. 